\title{
Correction to: A Finite Element/Operator-Splitting Method for the Numerical Solution of the Two Dimensional Elliptic Monge-Ampère Equation
}

\author{
Roland Glowinski ${ }^{1,2} \cdot$ Hao Liu $^{3}$ (D) Shingyu Leung ${ }^{3}$. Jianliang Qian ${ }^{4}$ \\ Published online: 28 October 2018 \\ (c) Springer Science+Business Media, LLC, part of Springer Nature 2018

\section{Correction to: Journal of Scientific Computing https://doi.org/10.1007/s10915-018-0839-y}

The article "A Finite Element/Operator-Splitting Method for the Numerical Solution of the Two Dimensional Elliptic Monge-Ampère Equation", written by Roland Glowinski, Hao Liu, Shingyu Leung and Jianliang Qian, was originally published electronically on the publisher's internet portal (currently SpringerLink) on 27 September 2018 with open access. With the author(s)' decision to step back from Open Choice, the copyright of the article changed on 3 October 2018 to (C) Springer Science+Business Media, LLC, part of Springer Nature 2018, and the article is forthwith distributed under the terms of copyright.

The original article can be found online at https://doi.org/10.1007/s10915-018-0839-y.

Hao Liu

hliuas@ust.hk

Roland Glowinski

roland@math.uh.edu

Shingyu Leung

masyleung@ust.hk

Jianliang Qian

qian@math.msu.edu

1 Department of Mathematics, University of Houston, 4800 Calhoun Road, Houston, TX 77204, USA

2 Department of Mathematics, The Hong Kong Baptist University, Kowloon Tong, Hong Kong

3 Department of Mathematics, Hong Kong University of Science and Technology, Clear Water Bay, Hong Kong

4 Department of Mathematics, Michigan State University, East Lansing, MI 48824, USA 